Marco Vinicio Guamán Alarcón

\title{
Previsão do efeito das trincas curtas em componentes estruturais
}

Dissertação apresentada como requisito parcial para obtenção do grau de Mestre pelo Programa de Pós-graduação em Engenharia Mecânica da PUC-Rio

Orientador: Prof. Jaime Tupiassú Pinho de Castro 


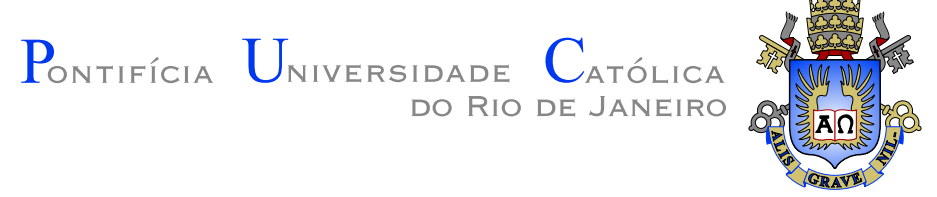

Marco Vinicio Guamán Alarcón

\section{Previsão do efeito das trincas curtas em componentes estruturais}

Dissertação apresentada como requisito parcial para obtenção do grau de Mestre pelo Programa de Pós-graduação em Engenharia Mecânica do Centro Técnico Científico da PUC-Rio. Aprovada pela Comissão Examinadora abaixo assinada.

Prof. Marco Antonio Meggiolaro

Departamento de Engenharia Mecânica — PUC-Rio

Prof. Paulo Pedro Kenedi

Centro Federal de Educação Tecnológica - CEFET-RJ

Prof. José Eugenio Leal

Coordenador Setorial do Centro Técnico Científico - PUC-Rio

Rio de Janeiro, 11 de Setembro de 2012 
Todos os direitos reservados. É proibida a reprodução total ou parcial do trabalho sem autorização da universidade, do autor e do orientador.

\section{Marco Vinicio Guamán Alarcón}

Graduou-se em Engenharia Mecânica em 2009, na Escuela Politécnica Nacional (Quito, Equador).

Ficha Catalográfica

Guamán, Marco Vinicio

Previsão do efeito das trincas curtas em componentes estruturais / Marco Vinicio Guamán Alarcón; orientador: Jaime Tupiassú Pinho de Castro. - Rio de Janeiro : PUC-Rio, Departamento de Engenharia Mecânica, 2012.

v., 93 f: il. ; $29,7 \mathrm{~cm}$

1. Dissertação (mestrado) - Pontifícia Universidade Católica do Rio de Janeiro, Departamento de Engenharia Mecânica.

Inclui referências bibliográficas.

1. Engenharia Mecânica - Tese. 2. Trinca curta. 3. Limiar de propagação. 4. Vida à fadiga. 5. Tamanho característico de trinca curta. 6. Modelagem das trincas curtas. 7. Fadiga. I. Castro, Jaime Tupiassú Pinho de. II. Pontifícia Universidade Católica do Rio de Janeiro. Departamento de Engenharia Mecânica. III. Título. 
A DIOS y a mi Patria A mis padres, Anita y José A mis hermanos, Darwin, José, Mary, Tomás y Fernanda $\mathrm{Al}$ amor de mi vida, Patty 


\section{Agradecimentos}

Ao Professor Jaime Tupiassú Pinho de Castro pelo apoio moral como amigo e pelo suporte como orientador no desenvolvimento deste trabalho.

Ao Professor Marcos Henrique pela ajuda na análise microscópica no Laboratório de Microscopia do DEMa.

À CAPES e à PUC-Rio pelos auxílios concedidos durante o tempo de desenvolvimento desta pesquisa.

À Patty pelo amor incondicional, pela paciência e pela colaboração na redação deste trabalho.

Ao Lucho por sua grande ajuda na minha fase inicial na PUC-Rio.

Ao meu amigo Byron "el compadre" por sua amizade incondicional.

Aos meus amigos do Laboratório de Fadiga, em especial ao Gerardo, pela amizade e ajuda proporcionada.

Aos meus familiares e amigos equatorianos que sempre mantiveram o contato e me apoiaram ao longo destes dois anos. Obrigado por acreditar em mim. 


\section{Resumo}

Guamán, Marco Vinicio; Castro, Jaime Tupiassú Pinho de (Orientador). Previsão do efeito das trincas curtas em componentes estruturais. Rio de Janeiro, 2012. 93p. Dissertação de Mestrado - Departamento de Engenharia Mecânica, Pontifícia Universidade Católica do Rio de Janeiro.

A resistência à fadiga dos elementos estruturais pode ser limitada pela presença de trincas curtas, as quais podem passar despercebidas em inspeções não-destrutivas tradicionais. Para modelar o comportamento dessas trincas pode-se utilizar o tamanho característico das trincas curtas $a_{0}$, proposto por El Haddad, Topper e Smith (ETS), que permite ajustar adequadamente os dados experimentais do diagrama de Kitagawa-Takahashi. Partindo do modelo ETS, neste trabalho se apresenta o modelo do Gradiente de Tensão (GT), desenvolvido para prever o efeito das trincas curtas que nascem em pontas de entalhes. Este modelo reconhece que trincas podem ser facilmente geradas por fadiga em entalhes afiados, que introduzem efeitos de concentração de tensão elevados nas suas pontas. Mas devido ao alto gradiente de tensão que atua em torno das pontas desses entalhes, as trincas curtas que deles emanam também podem parar de crescer por fadiga após terem se propagado por uma pequena distância, tornando-se assim trincas não-propagantes que podem ser toleradas em serviço. Corpos de prova tipo $\mathrm{C}(\mathrm{T})$ modificados foram projetados especificamente para estudar a geração e o crescimento inicial dessas trincas em ensaios de fadiga, e usados para verificar experimentalmente as previsões do modelo proposto.

\section{Palavras-chave}

Trinca curta; Limiar de propagação; Vida à fadiga; Tamanho característico de trinca curta; Modelagem das trincas curtas; Fadiga. 


\section{Abstract}

Guamán, Marco Vinicio; Castro, Jaime Tupiassú Pinho de (Advisor). Prediction of the short cracks effect on structural components. Rio de Janeiro, 2012. 93p. MSc Dissertation Departamento de Engenharia Mecânica, Pontifícia Universidade Católica do Rio de Janeiro.

The fatigue strength of structural elements may be limited by the presence of short cracks, which can not be detected with traditional nondestructive inspections. The behavior of these cracks can be modeled using the characteristic size of short cracks $a_{0}$, proposed by El Haddad, Topper and Smith (ETS), which allows one to properly adjust experimental data from the Kitagawa-Takahashi diagram. Based on the ETS model, this work presents the Stress Gradient (SG) model, developed to predict the effect of short cracks that grow from the notch tips. This model considers that cracks are easily generated due to fatigue in sharp notches, which introduce high stress concentration effects at their tips. Because of the high stress gradient acting at these notch tips, the short cracks can also stop to grow by fatigue after having propagated through a small distance, thereby becoming non-propagating cracks that can be tolerated in service. $\mathrm{C}(\mathrm{T})$ modified specimens were specifically designed to study the generation and initial growth of these cracks in fatigue tests, and used to experimentally verify the predictions of the proposed model.

\section{Keywords}

Short crack; Growth threshold; Fatigue stress limit; Fatigue short crack threshold; Short crack modeling; Fatigue. 


\section{Sumário}

1 Introdução $\quad 15$

$\begin{array}{lll}1.1 & \text { Visão global } & 16\end{array}$

$\begin{array}{lll}1.2 & \text { Objetivos } & 16\end{array}$

$\begin{array}{lll}1.3 & \text { Resultados esperados e limitações } & 17\end{array}$

$\begin{array}{lll}1.4 & \text { Organização da dissertação } & 17\end{array}$

2 Fundamentos teóricos $\quad 19$

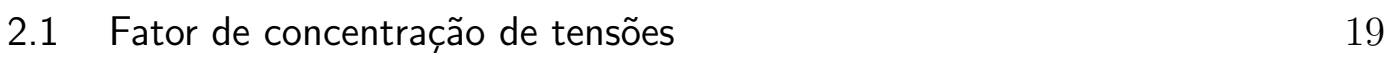

2.2 Fator de concentração de tensões em fadiga 21

2.3 Concentração de tensões em torno de uma trinca 23

2.4 Estimativa de Creager e Paris 24

2.5 O limiar de propagação de trincas curtas e o modelo ETS 26

2.6 O modelo GT 28

2.7 O modelo TCD 32

2.8 Trabalhos existentes sobre trincas curtas 34

3 Projeto dos corpos de prova e execução de ensaios de fadiga $\quad 35$

3.1 Seleção do material para fabricação dos CPs 35

3.2 Seleção da geometria do corpo de prova 36

3.3 Análise por Elementos Finitos 38

3.4 Aplicação do modelo GT a um corpo de prova tipo C(T) 40

3.5 Análise de sensibilidade do modelo GT 45

3.6 Aplicação do modelo TCD a um corpo de prova tipo C(T) 50

3.7 Ensaios múltiplos $\quad 52$

3.8 Fabricação e preparação dos corpos de prova 54

3.9 Ensaio a fadiga dos CPs $\quad 55$

3.10 Procedimento de monitoramento detecção das trincas $\quad 57$

$4 \quad$ Análise e comparação de resultados numéricos e experimentais obtidos $\quad 59$

4.1 Comparação dos valores numéricos determinados pelos modelos GT e TCD 59

$\begin{array}{ll}4.2 & \text { Ensaios a fadiga }\end{array}$

4.3 Análise por microscopia ótica 64

4.4 Comparação dos resultados experimentais com os calculados pelos modelos GT e TCD 64

4.5 Outros CPs para visualizar trincas não-propagantes 69

5 Conclusões e recomendações $\quad 75$

$\begin{array}{lll}5.1 & \text { Conclusões } & 75\end{array}$

5.2 Sugestões para continuar o trabalho 76

$\begin{array}{ll}\text { Referências Bibliográficas } & 77\end{array}$

A Paper apresentado no $67^{\text {th }}$ ABM International Congress $\quad 80$ 


\section{Lista de figuras}

2.1 Placa de Inglis [1] 20

2.2 Algumas medidas experimentais de $\mathrm{Kf}[10] \quad 22$

2.3 Trincas não-propagantes geradas se $S_{L}^{\prime} / K_{t}<\sigma_{n}<S_{L}^{\prime} / K_{f}$ [12]. 23

2.4 Modo I de carregamento de trincas [1]. 24

2.5 Alguns FIT catalogados na literatura [1]. 25

2.6 Coordenadas de Creager-Paris [1] 25

2.7 Diagrama de Kitagawa-Takahashi que descreve a propagação de fadiga de trincas curtas e longas sob cargas pulsantes $(R=0)$ num aço $\mathrm{HT} 80$ com $\Delta K_{0}=11.2 \mathrm{MPa} \sqrt{ } \mathrm{m}$ e $\Delta S_{0}=575 \mathrm{MPa}$.

2.8 Razão entre limiares de propagação de trincas curtas e trincas longas como função da razão $a / a_{0}$ [1].

2.9 Curvas de ajuste por Elementos Finitos (EF) gerados no Quebra2D e comparação com os resultados experimentais obtidos por Nishitani e Tada [1].

2.10 Geometria do corpo de prova de flexão em quatro pontos utilizado por [6] no seus experimentos.

3.1 Geometria de um corpo de prova tipo $\mathrm{C}(\mathrm{T})$ modificado.

3.2 Malha gerada no Abaqus para a análise por EF num corpo de prova tipo $\mathrm{C}(\mathrm{T})$, para valores de $b=15 \mathrm{~mm}$ e $\rho=1 \mathrm{~mm}$.

3.3 Tipos de carregamento analisados por EF no corpo de prova tipo $\mathrm{C}(\mathrm{T})$.

3.4 Gradiente de tensão à frente da ponta do entalhe, obtido por EF para um $\mathrm{C}(\mathrm{T})$, quando $b=15 \mathrm{~mm}$ e $\rho=1 \mathrm{~mm}$.

3.5 Coeficiente de fadiga FR versus teor de carbono para aços.

3.6 Esquema de curvas $\sigma_{a} \sigma_{m}$ típicas [1].

3.7 Funções adimensionais $f(a)$ e $g\left(a, \Delta S_{0} / \Delta \sigma_{n}, \Delta K_{0} / \Delta S_{0}, \gamma\right)$ para valores de $\rho=0.5 \mathrm{~mm}$ e $\Delta \sigma_{n}=46.78 \mathrm{MPa}$, respectivamente.

3.8 Fator de concentração de tensão linear elástico $K_{t}$ e fator de concentração de tensão em fadiga $K_{f}$, determinado pelo modelo GT, em função do raio da ponta do entalhe $\rho$, para $b=15 \mathrm{~mm}$.

3.9 Limiar de propagação $\Delta K_{t h}$ e faixa da gama do FIT onde se teria trincas não-propagantes em função do tamanho da trinca $a$, para $\rho=0.1 \mathrm{~mm}$.

3.10 Limiar de propagação $\Delta K_{t h}$ e faixa da gama do FIT onde se teria trincas não-propagantes em função do tamanho da trinca $a$, para $\rho=1 \mathrm{~mm}$.

3.11 Previsão da maior trinca não-propagante $a_{\max }$ em função do radio da ponta $\rho$ do entalhe, para $b=15 \mathrm{~mm}$.

3.12 Influência do valor do parâmetro de Bazant $\gamma$ no valor de $K_{f}$.
3.13 Influência do valor de $\Delta S_{0}$, estimado por Gerber, Goodman e Soderberg, no valor de $K_{f}$.

3.14 Relação entre $\Delta K_{t h} / S_{R}$ e $R$ para aços [23].

3.15 Limiar de propagação $\Delta K_{t h}$ versus razão de tensão $\mathrm{R}$ para alguns aços [24]. 
3.16 Influência do valor do limiar de propagação de trincas longas $\Delta K_{0}$ no valor de $K_{f}$.

3.17 Influência do valor de $\gamma$ no valor de $a_{\max }$.

3.18 Influência do valor de $\Delta S_{0}$,estimado por Gerber, Goodman e Soderberg, no valor de $a_{\max }$.

3.19 Influência do valor do limiar de propagação de trincas longas $\Delta K_{0}$ no valor de $a_{\max }$.

3.20 Fator de concentração de tensão linear elástico $K_{t}$ e fator de concentração de tensão em fadiga $K_{f}$ em função do radio da ponta do entalhe $\rho$, para $b=15 \mathrm{~mm}$.

3.21 Configuração de um $\mathrm{C}(\mathrm{T})$ modificado para múltiplos ensaios.

3.22 Fator de concentração de tensão linear elástico $K_{t}$ e fator de concentração de tensão na fadiga $K_{f}$ em função do radio do furo $\rho$, para $b=38 \mathrm{~mm}$.

3.23 Direção do lixamento das faces do CP.

3.24 Esquema da usinagem de um novo entalhe no $C(T)$.

3.25 Equipamento de ensaio marca INSTRON 8501.

3.26 Lente de aumento óptico utilizado para visualização do aparecimento de trincas.

3.27 Microscópio óptico utilizado para visualização de eventuais trincas não-propagantes.

4.1 Comparação dos $K_{f}$ determinados pelos modelos GT e TCD e o $K_{t}$ estimado por Creager-Paris, para um C(T) com $b=15 \mathrm{~mm}$.

4.2 Comparação dos $K_{f}$ determinados pelos modelos GT e TCD e o $K_{t}$ estimado por Creager-Paris, para um C(T) com $b=22.5 \mathrm{~mm}$.

4.3 Comparação dos $K_{f}$ determinados pelos modelos GT e TCD e o $K_{t}$ estimado por Creager-Paris, para um C(T) com $b=28.5 \mathrm{~mm}$.

4.4 Comparação dos $K_{f}$ determinados pelos modelos GT e TCD e o $K_{t}$ estimado por Creager-Paris, para um C(T) com $b=38 \mathrm{~mm}$.

4.5 Micrografia do entalhe com $b=38 \mathrm{~mm}$ e $\rho=0.62 \mathrm{~mm}$ do CP1, aumento 20X. a) ensaio prévio ao inicio da trinca, b) inicio e propagação de uma trinca.

4.6 Micrografia do entalhe com $b=22.5 \mathrm{~mm}$ e $\rho=1 \mathrm{~mm}$ do CP2, aumento $10 \mathrm{X}$. a) ensaio prévio ao inicio da trinca, b) inicio e propagação de uma trinca.

4.7 Micrografia do entalhe com $b=28.5 \mathrm{~mm}$ e $\rho=0.62 \mathrm{~mm}$ do CP2, aumento 10X. a) ensaio prévio ao inicio da trinca, b) inicio e propagação de uma trinca.

4.8 Micrografia do entalhe com $b=15 \mathrm{~mm}$ e $\rho=1 \mathrm{~mm}$ do CP1, aumento 10X. a) ensaio prévio ao inicio da trinca, b) inicio e propagação de uma trinca.

4.9 Valores numéricos de $K_{f}$ determinados pelos modelos GT e TCD em função do $K_{f}$ experimental. Pelo modelo GT se analisam três casos: $\Delta S_{0}$ calculado por Goodman, por Soderberg e $\Delta K_{0}=$ $12 \mathrm{MPa} \sqrt{ } \mathrm{m}$.

4.10 Zonas determinadas pelo modelo GT em que, segundo Frost, se deveriam ter trincas não-propagantes e comparação com resultados experimentais. 
4.11 Zonas determinadas com $\Delta S_{0}$ estimado por Soderberg pelo modelo GT em que, segundo Frost, se deveriam ter trincas não-propagantes e comparação com resultados experimentais.

4.12 Zonas determinadas com $\Delta K_{0}=12 \mathrm{MPa} \sqrt{ } \mathrm{m}$ pelo modelo GT em que, segundo Frost, se deveriam ter trincas não-propagantes e comparação com resultados experimentais.

4.13 Geometria e dimensões em milímetros do CP tipo SEN(T) modificado. 70

4.14 Comparação dos valores numéricos de $K_{f}$ obtidos pelos modelos GT e TCD e o $K_{t}$ calculado por Inglis, para um CP tipo SEN(T), quando $b=15 \mathrm{~mm}$.

4.15 Comparação dos valores numéricos de $K_{f}$ obtidos pelos modelos GT e TCD e o $K_{t}$ calculado por Inglis, para um CP tipo SEN(T), quando $b=50 \mathrm{~mm}$.

4.16 Geometria e dimensões em milímetros do CP tipo SE(B) modificado. 72

4.17 Comparação dos valores numéricos de $K_{f}$ determinados pelos modelos GT e TCD e o valor de $K_{t}$ estimado por Creager-Paris, para um CP tipo $\mathrm{SE}(\mathrm{B})$, quando $b=15 \mathrm{~mm}$.

4.18 Comparação dos valores numéricos de $K_{f}$ determinados pelos modelos GT e TCD e o valor de $K_{t}$ estimado por Creager-Paris, para um CP tipo $\mathrm{SE}(\mathrm{B})$, quando $b=30 \mathrm{~mm}$.

4.19 Comparação da maior trinca não-propagante $a_{\max }$ em função do raio $\rho$ da ponta do entalhe para corpos de prova tipo $\mathrm{C}(\mathrm{T}), \mathrm{SEN}(\mathrm{T})$ e $\mathrm{SE}(\mathrm{B})$ modificados, para uma valor $b=15 \mathrm{~mm}$. 


\section{Lista de tabelas}

3.1 Estimativas do tamanho característico de trinca curta $a_{0}$ para alguns aços estruturais [1].

3.2 Propriedades mecânicas do aço comercial ASTM A36. 36

3.3 Propriedades $\varepsilon N$ do aço ASTM A36 36

4.1 Resultados do modelo GT para um CP tipo $\mathrm{C}(\mathrm{T})$, sem considerar o efeito da tensão fletora causada pela carga $\Delta P(b=15 \mathrm{~mm}) . \quad 62$

4.2 Programa de teste para o entalhe número 1 do $\mathrm{CP} 1(b=15 \mathrm{~mm}) . \quad 63$

4.3 Resultados corrigidos para um CP tipo $\mathrm{C}(\mathrm{T})$, considerando o efeito da tensão fletora causada pela carga $\Delta P(b=15 \mathrm{~mm}) . \quad 63$

4.4 Resumo dos ensaios que permitiram a detecção de trincas. 64

4.5 Valores numéricos de $K_{f}$ determinados pelos modelos GT e TCD e erros relativos ao $K_{f}$ determinado experimentalmente. 


\section{Sumário das notações}

\section{Abreviaturas}

AM método da área do modelo TCD (area method)

$\mathrm{C}(\mathrm{T}) \quad$ corpo de prova compacto de tensão (compact tension specimen)

CP corpo de prova

C-P Creager e Paris

EF elementos finitos

ETS El Haddad, Topper e Smith

FIT fator de intensidade de tensões

FR coeficiente de fadiga (fatigue ratio)

GT gradiente de tensão

K-T diagrama de Kitagawa-Takahashi

LM método da linha do modelo TCD (line method)

MFLE mecânica da fratura linear elástica

PM método do ponto do modelo TCD (point method)

$\mathrm{SE}(\mathrm{B}) \quad$ corpo de prova de flexão (single-edge-notched bending specimen)

$\mathrm{SEN}(\mathrm{T})$ corpo de prova de tensão (single-edge-notched tension specimen)

SN método de dimensionamento contra a iniciação de trincas por fadiga (análise elástica)

TCD teoria da distância crítica (theory of critical distance)

VM método do volume do modelo TCD (volume method)

$\varepsilon \mathrm{N}$ método de dimensionamento contra a iniciação de trincas por fadiga (análise elastoplástica) 


\section{Símbolos}

a tamanho de trinca $[\mathrm{mm}]$

$a_{0} \quad$ tamanho característico de trinca curta $[\mu \mathrm{m}]$

$b \quad$ profundidade de entalhe [mm]

$C(\%)$ teor de carbono no aço

$F^{2} \quad$ fator de correlação de ajuste de dados

$f(a) \quad$ função adimensional que quantifica o gradiente de tensões à frente da ponta do entalhe

$f(a / W)$ função adimensional de geometria do fator de intensidade de tensão

$K_{f} \quad$ fator de concentração de tensão na fadiga

$K_{I} \quad$ fator de intensidade de tensão em modo I $[\mathrm{MPa} \sqrt{ } \mathrm{m}]$

$K_{t} \quad$ fator de concentração de tensão linear elástico

$L \quad$ distância crítica no modelo TCD $[\mu \mathrm{m}]$

$l_{r} \quad$ comprimento do ligamento residual de uma peça $[\mathrm{mm}]$

$q \quad$ sensibilidade ao entalhe

$R \quad$ razão de tensão $\sigma_{\min } / \sigma_{\max }$ ou $K_{\min } / K_{\max }$

$S_{E} \quad$ resistência ao escoamento [MPa]

$S_{F}(N)$ resistência à fadiga para um número de ciclos $\mathrm{N}[\mathrm{MPa}]$

$S_{L} \quad$ limite de fadiga da peça $[\mathrm{MPa}]$

$S_{L}^{\prime} \quad$ limite de fadiga do material $[\mathrm{MPa}]$

$S_{R} \quad$ resistência à ruptura [MPa]

$t \quad$ espessura da peça $[\mathrm{mm}]$

$W \quad$ largura da peça [mm]

$\alpha \quad$ fator de superfície livre do fator de intensidade de tensão

$\gamma \quad$ expoente de Bazant de ajuste de dados

$\mu \quad$ símbolo de micro $\left(10^{-6}\right)$

$\rho \quad$ raio de ponta do entalhe $[\mathrm{mm}]$

$\sigma_{a} \quad$ componente alternada da tensão [MPa]

$\sigma_{m} \quad$ componente média da tensão $[\mathrm{MPa}]$

$\sigma_{\max } \quad$ tensão máxima $[\mathrm{MPa}]$

$\sigma_{n} \quad$ tensão nominal $[\mathrm{MPa}]$

$\Delta K_{0} \quad$ limiar de propagação de trinca para $R=0[\mathrm{MPa} \sqrt{ } \mathrm{m}]$

$\Delta K_{I} \quad$ gamma do fator de intensidade de tensão em modo I [MPa $\left.\sqrt{ } \mathrm{m}\right]$

$\Delta K_{t h} \quad$ limiar de propagação de trinca $[\mathrm{MPa} \sqrt{ } \mathrm{m}]$

$\Delta P \quad$ gamma da carga aplicada $[\mathrm{kN}]$

$\Delta S_{0} \quad$ gamma do limite de fadiga do material para $R=0[\mathrm{MPa}]$

$\Delta \sigma \quad$ gama da tensão atuante no ponto crítico da peça [MPa]

$\Delta \sigma_{n} \quad$ gamma das tensões nominais [MPa] 